\title{
On the Link between Rotation and lithium in Giant stars
}

J. R. De Medeiros, J. D. Nascimento Jr, S. Sankarankutty, J. M. Costa, J. R. P. Da Silva and M. R. G. Maia

Universidade Federal do Rio Grande do Norte, Departamento de Física, 59072-970, Natal-RN, Brazil

\section{Introduction}

Late-type evolved stars are well known for the decrease with age of their rotation and lithium abundance. However, the root cause of this property as well as the relationship between rotation and lithium content in these stars are not yet completely established. In the present work, we study the link between rotation and lithium abundances in solar-type giant stars on the basis of a large sample of 380 stars of spectral type F, G and K.

\section{Observational data}

The data sample selected for the present investigation has as main characteristics the high precision of the rotational velocity and lithium abundance, as well as the large size of the sample.

Lithium abundances were taken from the following sources: $36 \mathrm{~F}$ and early$\mathrm{G}$ stars from Wallerstein et al. (1994), $10 \mathrm{~F}$ stars from Balachandran (1990) and 334 late-G and $\mathrm{K}$ type giants from Brown et al. (1989). Rotational velocities were taken from De Medeiros and Mayor (1999).

\section{Results}

Figure 1 shows the behavior of lithium abundance as a function of rotational velocity for the single and binary stars of the present sample.

One observes that giant stars presenting the highest lithium content, typically stars earlier than the spectral type G0III, are also those with the greatest rotation rate. Stars located to the right of the drop in rotation, namely stars later than G0III, present as a rule, the lowest rotation rate and lithium content.

Such features indicate a trend leading to a correlation between rotation and lithium abundance along the giant branch. An additional important feature is the large spread in lithium abundance for low rotators. Stars with a $v \sin i$ lower than about $4.0 \mathrm{~km} \mathrm{~s}^{-1}$ show a wide range of lithium abundance values with $\log n(\mathrm{Li})$ ranging from about -1.5 to the cosmic value, namely five orders of magnitude. Whereas, except for a few stars with $4.0<v \sin i<20.0 \mathrm{~km} \mathrm{~s}^{-1}$, most of the stars with enhanced rotation show an abundance of lithium around the cosmic value. 


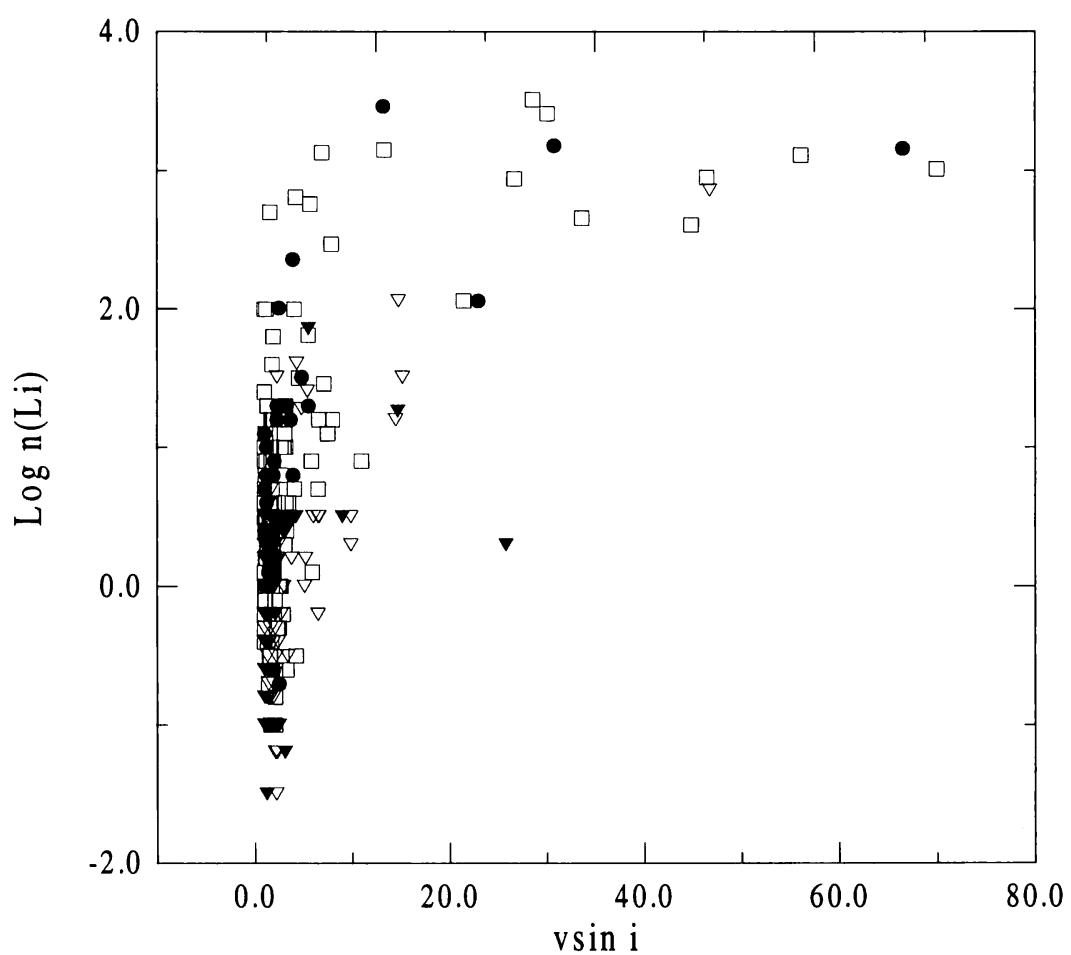

Figure 1. Lithium abundance as a function of rotational velocity (in units of $\mathrm{km} \mathrm{s}^{-1}$ ). Single stars are given as open squares while single stars with upper limit of $\mathrm{Li}$ abundances are represented by open triangles; binary systems are shown as filled circles whereas binary systems with upper limit of $\mathrm{Li}$ abundances are shown as filled triangles.

\section{References}

Balachandran S., 1990, ApJ 93, 235

Brown J. A., Sneden C., Lambert D. L., Dutchover Jr. E., 1989, ApJS 71, 293

De Medeiros J. R., Mayor M., 1999, A\&AS, 139, 433

Wallerstein G., Böhm-Vintense E., Vanture A. D., Gonzalez G., 1994, AJ 107, 2211 